\title{
DETERMINISTIC AND PROBABILISTIC REASONS AND CAUSES
}

\section{INTRODUCTION}

Ever since I first, as a student, encountered the distinction "Vernunftgrund Seinsgrund" in Stegmüller (1969), ch.II, I have been attracted and mystified by it. This essay is an attempt to get clearer about the mystery.

In (1969), ch.II, Stegmüller discusses the so-called thesis of the structural identity of explanation and prediction, which consists of two sub-theses, "namely (i) that every adequate explanation is potentially a prediction ...; (ii) that conversely every adequate prediction is potentially an explanation." (Hempel (1965), p. 367.) He lists a number of minor objections to this thesis which he thinks may be answered by more precise formulations of the thesis. But other objections suggest that there is something seriously wrong with sub-thesis (ii): a prediction needs only be based on Vernunftgründe, whereas an explanation cannot be content with Vernunftgründen, it must supply Seinsgründe; and therefore not every adequate prediction is potentially an explanation. Undoubtedly, this conclusion is convincing. ${ }^{1}$

Of course, a Seinsgrund, causa fiendi or essendi, is in this case nothing but a cause, and a Vernunftgrund, causa cognescendi, is nothing but a reason (to believe something ${ }^{2}$ ). But the old nomenclature has a bewildering appeal; it suggests a close analogy between Vernunftgründen and Seinsgründen without really hinting at where the similarity might be found.

Here is a preliminary picture of what I take the essence of this analogy to be. It seems clear that, if $A$ is a Grund for $B$, it is so only by virtue of a given background. This suggests that the similarity between Vernunftgründen and Seinsgründen consists in their having a common logical structure, because of which they are Gründe, and that their difference consists in their referring to different backgrounds. The background of Vernunftgründen seems to be clear: if $A$ is, at present, a Vernunftgrund for $B$ for 
me, the relevant background consists just of my present beliefs. But what might be the background of a Seinsgrund? Well, if $A$ is nature's Grund for $B$, could you think of a better background for this than all that nature has been up to $B$ - with the exception of $A$, of course? That is, wouldn't you agree that, if even the whole past of $B$ with the exception of $A$ does not provide a background showing $A$ to be nature's Grund for $B$, then $A$ simply lacks such a background, i.e., is not nature's Grund for $B$ ?

In what is to follow I try to make precise and defend this preliminary picture. In section 2, I shall give a standard account of what reasons are with the help of conditional logic and probability theory. In section 3, I shall summarize conditional, counterfactual analyses of causation, which seem to be the most preferred among philosophers. In section 4 , finally, an investigation of the ways in which reasons might fail to be causes and causes might fail to be reasons leads to an explication of causes which is very similar to, but more precise than that of section 3 .

In addition, I pursue a second aim. As is well known, the whole topic of causation, explanation and the like has been divided. It was dealt with either within a deterministic or within a probabilistic framework and often with little regard by the one framework to what was done within the other. One now definitely feels that the findings within the two frameworks can be acceptable only if there is an intimate connection between them or a common idea behind them. ${ }^{3}$ My intention is to lay stress upon what I think to be common to the two frameworks and to make clear that what is done within them easily flows from this common idea.

\section{REASONS}

I have two alarmclocks. That my first alarm is ringing gives me reason to expect that my second alarm will be ringing soon. The fact that my second alarm does not ring is evidence for me that it is broken. The fact that I thus overslept makes it very likely for me that I shall miss my appointment. And if I would believe in the axiom of choice, I would thereby believe in a well-ordering of the real numbers. So, what does a reason do? My belief in a reason strengthens my belief in what it is a reason for. Or to express it a bit more precisely:

(1) $\quad A$ is a reason for $B$ for the person $X$ at time $s$ iff $X$ 's believing $A$ at $s$ would raise the epistemic rank of $B$ for $X$ at $s$. 
As a starting point, (1) is good enough for us. ${ }^{4}$ But in order to make the statement of (1) still clearer, we have to say two things: what sort of things are $A$ and $B$, and what exactly is meant by an epistemic rank and its raising? We shall answer this by conventional, slightly technical methods.

Concerning the first question: We assume $W$ to be a set of possible worlds. As usual, any subset of $W$ is a proposition. Then, $A$ and $B$ are such propositions.

A digression about possible worlds and propositions: Unfortunately, these explanations still can't pass without commentary. I have to make three remarks.

As "possible world" seems persistently to be a problematic notion (for the simple reason that it is a rather misleading name for what most people, including me, mean by it), let me explicitly state that I mean by a possible world something which is completely specifiable by certain fixed linguistic resources, i.e., a state-description given in an object language or a metalinguistically specified model for an object language or the like; I do not mean by it something extralinguistic over and beyond this as does in particular David Lewis. ${ }^{5}$ This is a harmless and relatively clear understanding of possible worlds (as clear as the presupposed fixed linguistic resources). It does not give us a very good notion of a world, since our world is not supposed, by most of us, to be exhaustively describable by any given actual language or even by a future language. ${ }^{6}$ But it does give us a not very bad notion of possibility, and that's decisive for our acceptance of it.

Secondly, I obviously intend to take propositions as objects of our beliefs. This is more than questionable, as is well known. For this purpose, propositions are likely to be too poorly individuated; if not too poorly, then too coarsely individuated, i.e., it may be too hard for us to recognize that two sentences express the same proposition; and they are likely to be too eternal, i.e., too purified of contextual components. However, let us not complicate our topic by considering other, still controversial proposals of more appropriate epistemic objects; let us stick to propositions for the sake of simplicity.

Finally, although we are not yet dealing with causation, you may have already suspected that I am going to take propositions also as objects of causal relations. Otherwise the suggested analogy between reasons and 
causes would break down at the very beginning. Now Donald Davidson has argued powerfully ${ }^{7}$ that the logical form of causal statements is such that they relate events and not propositions. Thus, some comments seem to be in order.

A strong point against propositions as causal objects was provided by the old Frege-Quinean argument that intensionality, i.e. substitutability of logical equivalents salva veritate, plus referential transparency implies truth-functionality ${ }^{8}$ - when combined with the firm conviction that causal statements are referentially transparent, but never truth-functional. I share this conviction. But the Frege-Quinean argument may be easily escaped, I think: Let us define that the proposition de dicto expressed by a sentence " $F a$ " is the set of possible worlds $w \in W$ such that the individual denoted by " $a$ " in $w$ lies in the extension of " $F$ " in $w$, and that the proposition de re expressed by " $F a$ " is the set of possible worlds $w \in W$ such that the individual actually denoted by " $a$ " (in the real world) lies in the extension of " $F$ " in $w$. This distinction may be easily extended to all other sentences (of, say, first order predicate logic). ${ }^{9}$ Now, if epistemic objects are propositional at all, then, obviously, propostions de dicto are the objects of de dicto-beliefs, and propositions de re are the objects of de re-beliefs. And I think, propositions de re are also the appropriate objects of causal relations. If we take them thus, causal statements are referentially transparent, simply because " $F a$ " and " $F b$ " express the same proposition de re, if only " $a=b$ " is true. But they are not intensional! This is so because logically equivalent sentences usually, but not necessarily express the same proposition de re. One counter-example is provided just by the Frege-Quinean argument: Let " $p$ " express a contingent proposition de re, i.e., neither $W$ nor $\varnothing$. Then the sentence " $\{x \mid x=x \& p\}=\{x \mid x=x\}$ " is logically equivalent to " $p$ "; the proposition de re expressed by it, however, is not contingent, but either the necessary $W$, if " $p$ " is true, or the impossible $\varnothing$, if " $p$ " is false. Hence, the premises of the Frege-Quinean argument simply do not apply, if propositions de re are taken as causal objects, and one important reason for taking events as causal objects thus dissolves.

Rather, there is an important heuristic reason against taking events as causal objects. Perhaps we should first say what is meant by an event. The meaning is unsharp, but the most common and, in particular, Davidson's understanding seems to be that an event is a more or less well defined spatio-temporal section of our real world. Thus, the identity conditions of 
events are given by their spatio-temporal boundaries. ${ }^{10}$ This implies that events resemble the real world in that they may not be exhaustively describable by any fixed linguistic resources (though they are of course nameable like any other object). In this important sense, events are not entities derived from language, as opposed to propositions. Now, all the papers arguing or insisting that the proper logical form of causal statements consists in applying causal relations to events have a very astonishing feature: they hardly say anything about the nature of causal relations, how they might be analyzed or theoretically accounted for. ${ }^{11}$ On the contrary, it may be observed, and we shall have the opportunity to do so, that virtually all more or less successful attempts at an analysis or theory of causation take propositions or other entities derived from language as causal objects. This does not prove, but it suggests that the true, i.e., our best theory of causation will do so as well. And it seems very clear to me that arguments concerning the logical form of something are relevant only insofar as they are conducive to a good overall theory of that something, and that in the end we had best take those entities as causal objects with which we can build our best theory of causation.

So let us be content here with propositions, and let us henceforth talk plainly of propositions; the distinction between propositions de re and propositions de dicto will not be relevant to the rest of this paper. End of digression.

We may thus turn to the second question: What exactly is meant by the epistemic rank of a proposition $B$ for a person $X$ and by raising that rank? We shall become clearer about this, if we describe more precisely the epistemic apparatus of our person $X$. There are essentially two highly idealized standard ways of doing this. It is here that the technical division into a deterministic and a probabilistic framework takes place.

The first way is to describe $X$ 's beliefs or epistemic state qualitatively: $X$ may either believe a proposition $B$ or disbelieve it, i.e., believe not- $B$, or be neutral with respect to it, i.e., believe neither $B$ nor not- $B$. The accompanying idealized rationality assumptions are that the beliefs of $X$ are consistent and closed under logical entailment. ${ }^{12}$ This implies that there is a non-empty proposition $C$ such that, for all propositions $D, X$ believes $D$ iff $C \subseteq D$; of course, $C$ is equal to $\cap\{D \mid X$ believes $D\}$. Let us call $C$ the total of $X$ 's epistemic state. Within the deterministic framework, we may thus describe an epistemic state simply by its total. 
Secondly, we may describe $X$ 's epistemic state in a quantitative way: $X$ may believe $B$ to some degree between 0 and 1 . The accompanying idealized rationality assumption is that these degrees conform to the laws of mathematical probability. ${ }^{13}$

This answers the question about the possible epistemic ranks of propositions. It is also clear how the ranks are ordered within each of the two frameworks, i.e., which of two ranks is the higher one. By this, however, we do not yet have a description of the raising of epistemic ranks. In order to know whether $X$ 's belief in $A$ at $s$ would raise the epistemic rank of $B$ for $X$ at $s$, we have to compare two epistemic states of $X$ and the ranks $B$ has in each. The first epistemic state is the one which $X$ actually has at $s$, and the second is the one into which the first one changes or would change by adding at $s$ the belief in $A$. This is usually expressed by saying that the second epistemic state is the first conditionalized by $A$. Thus, we have still to give an account of such belief changes or conditionalizations of epistemic states.

Within the probabilistic framework, there is a standard account as old as probability theory itself: If the probability measure $P_{X, s}$ describes $X$ 's actual epistemic state at $s$, then $X$ 's epistemic state conditional on $A$ is given by $P_{X, S}(. \mid A)$ which assigns to every $B \subseteq W$ the conditional probability $P_{X, s}(B \mid A)=P_{X, s}(A \cap B) / P_{X, s}(A)$. This method of conditionalization is not completely general; it fails iff $P_{X, s}(A)=0$. But this gap does not seem to be very grave, since propositions of probability 0 are rare, so to speak. ${ }^{14}$ We may thus translate (1) into this:

If $A, B \subseteq W$ and $0<P_{X, s}(A)<1$, then $A$ is a reason for $B$ for $X$ at $s$ iff $P_{X, s}(B \mid A)>P_{X, s}(B \mid \bar{A})$ (where $\bar{A}$ is to be the relative complement $W \backslash A$ of $A) .{ }^{15}$

Within the deterministic framework, a standard account of conditionalization has emerged only in the last fifteen years. A plausible try consists in this first rule: If $X$ 's actual epistemic state at $s$ is characterized by its total $C$, then the total of $X$ 's epistemic state conditionalized by $A$ is $A \cap C$. This expresses the reasonable idea that by learning $A X$ adds to his old beliefs the belief in $A$ as well as all logical consequences of $A$ and his old beliefs. However, if that rule were to hold generally, then $X$ would have to believe everything after learning a proposition $A$ which he believed not to be the case, i.e., for which $C \subseteq \bar{A}$. Such a complete epistemic collapse is 
far too dramatic an effect. Thus, we should restrict this rule to the case where $A \cap C \neq \varnothing$, thereby opening a gap similar to the one above in the rule of probabilistic conditionalization. However, this gap is more serious, because learning something disbelieved is not so rare as learning something of subjective probability 0 . Therefore, we should close it. This may be done easily by the following, almost trivial, subsidiary rule: If $A \cap C=\varnothing$, then by learning $A$ the old total $C$ changes to some new total $C^{\prime}$ with $\varnothing \neq C^{\prime} \subseteq A$. This only says that in the new epistemic state conditional on $A X$ believes $A$ and satisfies the above rationality conditions. To say anything general beyond this about the epistemic state conditional on $A$ seems hardly possible.

Let us summarize this by defining:

A function $g$ from the power set of $W$ except $\varnothing$ into the power set of $W$ is a selection function $(\text { for } W)^{16}$ iff the following holds for all non-empty $A, B \subseteq W$ :

(i) $\varnothing \neq g(A) \subseteq A$,

(ii) if $g(A) \cap B \neq \varnothing$, then $g(A \cap B)=g(A) \cap B$.

And a relation $G$ is a conditional relation (for $W$ ) iff there is a selection function $g$ for $W$ such that for all $A, B \subseteq W G(B \mid A)$ iff $g(A) \subseteq B$. Instead of $G(B \mid W)$ we simply write $G(B)$.

We can now describe $X$ 's actual epistemic state at $s$ plus its conditionalities with a selection function $g_{X, s}$ or the conditional relation $G_{X, s}$ derived from $g_{X, s \cdot} g_{X, s}(W)$ is the total of $X^{\prime}$ 's actual epistemic state at $s$, and $G_{X, s}$ $(B)$ holds iff $X$ believes $B$ at $s$. Similarly, $g_{X, s}(A)$ is to be interpreted as the total of $X$ 's epistemic state at $s$ conditionalized by $A$, and $G_{X, s}(B \mid A)$ holds iff $X$ believes $B$ at $s$ conditional on $A$. Clause (i) of (2) secures that actual and conditional epistemic states are consistent and includes the above subsidiary rule. Clause (ii) generalizes our first rule: whereas that rule describes only the transition from the actual to a conditional epistemic state, clause (ii) generally describes the transition from an epistemic state conditional on some $A$ to its further conditionalization by some $B$ (the result of which is the epistemic state conditional on $A \cap B) .{ }^{17}$

Now, we can finally state also the deterministic translation of (1):

$$
\begin{aligned}
& \text { If } A, B \subseteq W \text { and } \varnothing \neq A \neq W \text {, then } A \text { is a reason for } B \text { for } X \text { at } \\
& s \text { iff }
\end{aligned}
$$


(i) $G_{X, s}(B \mid A)$, but not $G_{X, s}(B \mid \bar{A})$, or

(ii) $G_{X, s}(\bar{B} \mid \bar{A})$, but not $G_{X, s}(\bar{B} \mid A)$.

(1d) covers all possible raisings of the qualitative epistemic rank of $B$. In case (i) $B$ moves from "disbelieved" or "neutral" to "believed", whence we might say that $A$ is a sufficient reason for $B$ in this case; and in case (ii) $B$ moves from "disbelieved" to "neutral" or "believed", whence we might say that $A$ is a necessary reason for $B$ in that case. All other raisings are obviously definable by these two raisings.

Certainly, the more literal translation of (1) would have been to write “(iii) $G_{X, s}(B \mid A)$, but not $G_{X, s}(B)$, or (iv) $G_{X, s}(\bar{B} \mid \bar{A})$, but not $G_{X, s}(\bar{B})$ " instead of "(i) or (ii)". However, our formulation is slightly preferable. If $G_{X, s}(A)$ does not hold, then (i) and (iii) are equivalent. But, if $G_{X, s}(A)$ does hold, $A$ could not be a sufficient reason for $B$ according to (iii); it can be according to (i), in the sense that, if $A$ should turn out to be false, the belief in $B$ would also be lost. Hence, (i) seems to be more adequate than (iii). The corresponding remarks apply to the relation between (ii) and (iv). In section 4 this point will prove to be essential. ${ }^{18}$

\section{CAUSES}

After the decline of the regularity theory of causality, the only promising approach seems to be to try a counterfactual analysis of causation. ${ }^{19}$ This is a preliminary statement of it:

$A$ is a cause of $B$ iff

(i) $A$ and $B$ both obtain, and $A$ earlier than $B$,

(ii) if $A$ had not been the case, $B$ would not have been the case, either. ${ }^{20}$

Clause (i) is not to dogmatically exclude the possibilities of simultaneous or even backwards causation. Rather, the intent is only to leave aside these intricate possibilities and to restrict our discussion to the normal case, where the cause in fact precedes the effect; this normal case is difficult enough. Apart from this, it is hard to deny the correctness of (3d). ${ }^{21}$ However, this only means that the counterfactual is as much in need of explication as is the concept of causation. Still, some small improvements on (3d) are easily made. 
A first thing to be noted is that in (3d) $A$ and $B$ cannot be arbitrary propositions; rather, each has to refer to a definite point of time. ${ }^{22}$ For instance, if the possible worlds in $W$ represent all possible infinite series of throws of a certain die, then there are propositions referring to a definite time such as $\{w \in w \mid$ according to $w$, no six turns up in the fifth throw $\}$ and propositions not referring to a definite time such as $\{w \in W \mid$ according to $w$, no six turns up in the first ten throws $\}$. Only propositions of the first kind are potential causes and effects. Let us call them instantaneous propositions and denote them by $A_{t}, B_{t^{\prime}}, \ldots$, where $t, t^{\prime}, \ldots$ are the points of time referred to by $A_{t}, B_{t^{\prime}}, \ldots$ Of course, we also assume that the points of time $t, t^{\prime}, \ldots$ are ordered by the ordering "earlier than", symbolized by " $<$ ".

We are thus prepared for a reformulation of (3d):

$A_{t}$ is a cause of $B_{t^{\prime}}$ iff

(i) $A_{t}$ and $B_{t^{\prime}}$ both obtain, and $t<t^{\prime}$,

(ii) under the obtaining circumstances, $A_{t}$ is a necessary and/or sufficient condition for $B_{t^{\prime}}$.

(4d) is presumably the most widely accepted preliminary analysis of causation. ${ }^{23}$ It makes explicit two things hidden in (3d):

Whereas (3d), if taken literally, requires $A_{t}$ to be only a necessary condition for $B_{t^{\prime}}$, the ambiguous "and/or" of (4d) lays open the issue concerning whether causes are to be necessary conditions or sufficient conditions or both. ${ }^{24}$ I shall not attempt to decide this issue; perhaps the simplest solution is to distinguish between sufficient and necessary causes and not to try to decide which logical combination thereof represents causes simpliciter.

But what is more important, (4d) makes explicit the dependence of the cause-effect relation on the obtaining circumstances, which is only implicit in (3d). Let us consider an example. Standing at a crossing, we see the traffic light turn red and a car stop. Of course, we assume that the first is a cause of the second, and also that if the first had not happened, the second would not have happened, either. But we may be wrong. Perhaps the driver of the car was completely drunk and stopped only to leisurely decide which of the two lanes he sees might be the right one. Or it might have been a police car which was about to pass the crossing with hooting sirens, but has just received new orders. Thus, the truth or acceptability 
of the causal and the counterfactual assumption of ours depends upon the fact that the driver of the car was attentive, had no privileges, etc., i.e. upon the obtaining circumstances. And that's the way of causal and counterfactual statements in general, as Lewis (1973a), sect. 3.2, has rightly stressed and as (4d) reflects.

As obvious this point is, it is difficult to say what exactly are the obtaining circumstances of a given cause and its effect. In trying to do this one seems to run into two circularities: First, one may be tempted to count everything that happened before the cause as belonging to the obtaining circumstances. This is probably harmless, but, intuitively, this is much too much. Intuitively, one would include into the circumstances of our example such things as the given traffic situation at the crossing, the condition of the car and its driver, etc., but not, say, all the things Nessie has done up to now in Loch Ness. Thus, only the relevant facts belong to the obtaining circumstances, and without doubt, "relevant" means here "causally relevant". Secondly, some things being the case in the time between cause and effect must certainly be included among the obtaining circumstances - in our case, e.g., the fact that no hooting police car is approaching from behind which would cause our driver not to stop, but to make way, or standing conditions like the functioning of the braking system of our car. However, it is equally certain that some things being the case in the time between cause and effect must not belong to the obtaining circumstances - for example the fact that the driver has applied the brakes, since, given this fact, the light's turning red would not be a necessary or sufficient condition for the stopping of the car. This suggests that only those facts which do not causally depend on the cause can be admitted as belonging to the obtaining circumstances. But then, we are obviously caught in another circle. ${ }^{25}$

We shall return to the problems posed by these apparent circularities in section 4. For the moment, it is only important to recognize the essential role of the obtaining circumstances in an analysis of causation.

A further point which is probably tacitly understood in (4d) needs to be made explicit. Certainly, "under the obtaining circumstances, $A_{t}$ is a sufficient condition for $B_{t}$ " "is to mean not only "given the obtaining circumstances and $A_{t}, B_{t^{\prime}}$ is necessary", but also "given the obtaining circumstances and $\bar{A}_{t}, B_{t^{\prime}}$ is not necessary, i.e., contingent or impossible". Likewise, "under the obtaining circumstances, $A_{t}$ is a necessary condition for 
$B_{t}$ '" is to mean not only "given the obtaining circumstances and $\bar{A}_{t}, B_{t^{\prime}}$ is impossible", but also "given the obtaining circumstances and $A_{t}, B_{t}$ ' is not impossible, i.e., contingent or necessary". This is reflected in our next reformulation:

$A_{t}$ is a cause of $B_{t^{\prime}}$ iff

(i) $A_{t}$ and $B_{t^{\prime}}$ both obtain, and $t<t^{\prime}$,

(ii) under the obtaining circumstances, $A_{t}$ raises the modal rank of $B_{t^{\prime}}$.

Here, the ordering of modal ranks in the deterministic case naturally is, from below, "impossible", "contingent", and "necessary". And the problem about the "and/or" in (4d) is now transformed into the question which of the various possible raisings of modal rank is characteristic of causation.

By having driven our discussion to (5), we have reached a first conspicuous analogy between reasons and causes, i.e. between (1) and (5). We have also quietly left the deterministic framework. For, we might as well interpret the modal ranks of (5) as probabilities, as we have already done in (1), and then we might use (5) as quite a useful leading idea for an explication of probabilistic causality. I am well aware that the topic of probabilistic causality still seems to be rather absurd for a number of philosophers, but I won't argue now that it is not; this is done very convincingly in the introduction of Suppes (1970). ${ }^{26}$

We may thus make the same move as in section 2 . Then, (5) splits into this:

(5d) $\quad A_{t}$ is a cause of $B_{t^{\prime}}$ iff

(i) $A_{t}$ and $B_{t^{\prime}}$ both obtain, $t<t^{\prime}$, and:

(ii) under the obtaining circumstances, $G\left(B_{t^{\prime}}, \mid A_{t}\right)$, but not $G\left(B_{t^{\prime}} \mid \bar{A}_{t}\right)$, and/or

(iii) under the obtaining circumstances, $G\left(\bar{B}_{t^{\prime}} \mid \bar{A}_{t}\right)$, but not $G\left(\bar{B}_{t}, A_{t}\right)$.

(5p) $\quad A_{t}$ is a cause of $B_{t^{\prime}}$ iff

(i) $A_{t}$ and $B_{t^{\prime}}$ both obtain, $t<t^{\prime}$, and

(ii) under the obtaining circumstances, $P\left(B_{t^{\prime}} \mid A_{t}\right)>P\left(B_{t^{\prime}} \mid \bar{A}_{t}\right)$. 
The meaning of the clause "under the obtaining circumstances" in (5d) and (5p) is as unclarified as it was in (4d); I promised to return to this later on. There is yet another contrast to (1d) and (1p): " $G$ " and " $P$ " have lost their subscript " $X, s$ ". Is this important? Indeed; to explain why requires another interlude.

A digression about epistemic and realistic interpretations: In section 2, it was very clear that conditional relations and probability measures had to be interpreted as describing the epistemic state of some $X$ at some time $s$ (plus its conditionalities). For (5d) and (5p) this interpretation seems rather unwelcome. I don't want to deny this, but let me draw attention to the fact that it still makes sense to interpret the " $G$ " and the " $P$ " in (5d) and (5p) epistemically by affixing the subscript " $X, s$ " to them. Only the explicandum slightly changes thereby. (5d) and (5p) then explicate something like " $A_{t}$ is a cause of $B_{t^{\prime}}$ according to the conceptions of $X$ at $s$ ". But beware; you may not paraphrase this by " $X$ believes at $s$ that $A_{t}$ is a cause of $B_{t}$ ', because $X$ may not know at $s$ that $A_{t}$ and $B_{t^{\prime}}$ obtain and may have only partial or even wrong beliefs about the obtaining circumstances. Thus, what is explicated then is rather " $X$ would believe at $s$ that $A_{t}$ is a cause of $B_{t^{\prime}}$, if informed about the obtaining of $A_{t}$ and $B_{t^{\prime}}$ and the relevant circumstances".

But, as we said, we need another interpretation of conditional relations and probability measures which is less epistemically framed and relativized. With probability, we do not seem to have problems; just interpret it as objective or statistical probability, as chance or propensity, or what you like. What an objectified conditional relation might be, is, however, more difficult to tell. One idea might be to link it up with the true laws of nature: Let $L$ be the proposition that conjoins all laws of nature, and define $G_{L}$ by

$$
G_{L}(D \mid C) \text { iff }\left\{\begin{array}{ll}
L \cap C \subseteq D, & \text { if } L \cap C \neq \varnothing \\
C \subseteq D & \text { if } L \cap C=\varnothing
\end{array} \text { for all } C, D \subseteq W\right.
$$

Thus, $G_{L}$ represents nomological implication ${ }^{27}$, and it certainly gives quite a good conception of an objectified conditional relation (though the concept of a law is not so clear). However, it can't be completely correct, since 
with $G_{L}(5 \mathrm{~d})$ reduces to a regularity account of causation which seems ultimately untenable. A second idea might be to identify the " $G$ " of (5d) with one of the subjunctive conditionals for which realistic semantics have been formulated, provided these are found comprehensible. ${ }^{28}$

I have no further idea on what an objectified conditional relation might be. It should be pointed out, however, that probability measures are actually in no way better off than conditional relations. Rather, the thesis that the deterministic and the probabilistic framework are only two sides of one and the same coin fits here well-nigh perfectly:

On both sides of the coin, the subjectivistic, epistemic interpretation is the easy, unproblematic one. Concerning subjective probability, this is now commonly accepted. I would be glad, if everybody would also concede this with respect to the accounts of epistemically interpreted conditional relations which I have quoted in note 17.

On both sides, the objectivistic, realistic interpretation is obscure and controversial. I think, it is not claiming too much to say that every existing attempt to such an interpretation either shrinks back from full generality and concentrates on safer details or is not very intelligible in its generality and has more skeptics than adherents. I do not mean thereby to belittle these attempts, but certainly, our present state of knowledge still leaves much to be desired.

To be a bit more specific: On both sides, e.g., it is already unclear which propositions belong to the domain of a realistically interpreted $G$ or $P$. One generally feels that objective probability makes sense only within not too large confines, and analogously, one hesitates with many counterfactual or subjunctive statements to assign them any truth value at all. ${ }^{29}$ In contrast to this, one has no such difficulties with the epistemic interpretation on both sides.

On both sides, we have a very close connection between the single case and the long run. On the probabilistic side, long run frequencies usually are good evidence about single-case probabilities, and in many cases though it is hard to say which - we are safe to identify limiting relative frequency and single-case probability. On the deterministic side, observed regularities are often good reason to believe in counterfactuals, and we have many universal propositions - though it is hard to say which - that support the corresponding counterfactual or subjunctive statement about a single case. 
The list may be easily extended. Perhaps it is also worth mentioning that on both sides presumably all existing interpretations may be said, despite their many subtle differences, to be either epistemic subjectivistic or realistic objectivistic, without doing too much violence to them.

This strict similarity of the deterministic and the probabilistic side seems to me to be very important. It may help to avoid the wrong and yet infer the right ways on the one side, which have been realized on the other side; it may offer new ways of mutual confirmation and disconfirmation. Let me give an example. There is that beautiful (1980) paper of Lewis which deals with the connection between subjective and objective probability and in fact claims to capture all we know about objective probability by his Principal Principle. Now I think that essential parts of this paper may be rewritten with a " $G$ " instead of a " $P$ " 30 and that the new paper would be as reasonable, plausible, or implausible as the old one. The force of our analogy would then be that you should have the same attitude to both, the real and the fictional paper. ${ }^{31}$

The actual situation being as described, I will be excused, I hope, for backing out of a realistic interpretation of conditional relations. Still, the reason why it is so hard to find a satisfying realistic interpretation is not yet very clear. In my view, it ultimately amounts to a most central or, since Kant, perhaps the central problem of theoretical philosophy: namely the problem in what sense and to which extent reality is a projection of our epistemic make-up. History shows that any quick and ready solution to this problem is almost sure to be false. ${ }^{32}$. And I am convinced that a satisfying realistic interpretation of conditional relations and probability measures must go hand in hand with a satisfying solution to this eminent problem. End of digression.

\section{FROM REASONS TO CAUSES}

But we should return from these hazy heights to more concrete fields. Let us therefore leave these interpretational problems of (5); this section will be deliberately ambiguous in this respect, it can and should be read under both interpretations.

There were other problems with (5); there are still "the obtaining circumstances", which are troublesome. Let us tackle them in an indirect way, by a strategy which has been most clearly displayed by Suppes (1970). 
The first, rhetorical step of this strategy consists in equating causes with reasons. Thus define: $A_{t}$ is prima facie a cause of $B_{t^{\prime}}$ iff $A_{t}$ and $B_{t^{\prime}}$ both obtain, $t<$ $t^{\prime}$, and $A_{t}$ is a reason for $B_{t^{\prime}}$ (in the sense of (1d) or $1 \mathrm{p}$ )). ${ }^{33}$

The second, serious step is to think about what's so wrong with this equation. The idea is that the more completely we know the ways in which prima facie causes might fail to be causes and causes might fail to be prima facie causes, the better we know what causation is for us. I think that there are essentially three ways in which causes and prima facie causes may differ: a prima facie cause may turn out to be a spurious, i.e., not a cause; a prima facie cause may turn out to be only an indirect cause; and a cause may be hidden, i.e., is eventually not a prima facie cause. Let me explain this in more detail. ${ }^{34}$

First, consider the case of spurious causes. The classical example for this is provided by barometers. The fact that my barometer is falling quickly is a good reason for assuming that there will soon be a thunderstorm over Munich (you may construe this deterministically or probabilistically). Thus, the barometer's falling is prima facie a cause of the thunderstorm, but certainly not a cause. Why? Because it is also a fact that a low pressure area is rapidly approaching Munich, and because, given this fact, the behavior of my barometer does not constitute any reason for the thunderstorm. There are many similar examples. The common structure of such examples seems to be this:

We are given three true instantaneous propositions $A_{t}, B_{t^{\prime}}$, and $C_{t *}$ such that

(i) $t^{*}<t<t^{\prime}$,

(ii) $A_{t}$ is a reason and, thus, prima facie a cause for $B_{t^{\prime}}$,

(iii) given $C_{t *}, A_{t}$ is not a reason for $B_{t^{\prime}}$.

Then, and only then, we may say that $A_{t}$ is a spurious cause of $B_{t^{\prime}}$ because of $C_{t *}{ }^{35}$

Secondly, we have the case of indirect causes. An example for this has already been given. The fact that the traffic light turns red is a good reason for assuming, and thus prima facie a cause of the fact, that the car ap- 
proaching the light will stop. But, after the light has turned red, the driver of the car applies the brakes. Again, given this fact, the light's turning red is no longer a reason for the car's stopping. But this does not show that the light's turning red is not a cause of the car's stopping, it only shows that it is an indirect cause which is mediated by further facts, e.g., the fact that the driver has applied the brakes. The reason why we do not assume spurious causation in this case lies, of course, in the different temporal position of the third interfering fact, which is now between the prima facie cause and its prima facie effect. The general structure of this example may be described thus:

We are given three true instantaneous propositions $A_{t}, B_{t^{\prime}}$, and $C_{t *}$ such that

(i) $t<t^{*}<t^{\prime}$,

(ii) $A_{t}$ is a reason and, thus, prima facie a cause for $B_{t^{\prime}}$,

(iii) given $C_{t *}, A_{t}$ is not a reason for $B_{t^{\prime}}$.

Then, and only then, we may say that $A_{t}$ is an indirect cause of $B_{t^{\prime}}$ because of $C_{t *}{ }^{36}$

Thirdly, there is the case of hidden causes. I press a light switch, but, unexpectedly, the light does not go on. Thus, the former is prima facie not a cause of the latter. What has happened? Accidentally, another person has simultaneously pressed the other switch for that light. Then it becomes apparent, that my pressing my switch was a cause for the light's not going on. Another hypothetical example, taken from statistics, is "that we do not find a correlation between the amount of rainfall and the amount of wheat produced, measured over consecutive years, whereas the partial correlation after elimination of the effect of daily temperature is positive. That is, for years with equal temperature, there is a correlation between rainfall and amount of wheat produced, but this relation is contaminated by variation in temperature, since higher rainfall accompanies lower temperature, which is disadvantageous for wheat production." (Van de Geer (1971), p. 106f.) Thus, on statistical grounds, the fact that we just had such a wet year is in itself no reason for the rich wheat crop, but considering the fact that this year was still relatively warm, it is. Again, the general structure is obvious: 
We are given three true instantaneous propositions $A_{t}, B_{t^{\prime}}$, and $C_{t *}$ such that

(i) $t, t^{*}<t^{\prime}$,

(ii) $A_{t}$ is not a reason and, thus, prima facie not a cause for $B_{t^{\prime}}$,

(iii) given $C_{t *}, A_{t}$ is a reason for $B_{t^{\prime}}$.

Then, and only then, we may say that $A_{t}$ is a hidden cause of $B_{t^{\prime}}$ because of $C_{t *}{ }^{37}$

There are examples apparently showing the inadequacy of (7) - (9), most notably cases of asymmetric overdetermination or pre-emption. Consider the famous case of the desert traveller ${ }^{38}$, who has two enemies seeking his life. The first pours deadly poison into his water can $\left(A_{t}\right)$; later, but independently of this, the other drills a hole into the bottom of the can $\left(C_{t *}\right)$. Thus, the traveller has nothing to drink, and the sad end of the story is that he dies $\left(B_{t^{\prime}}\right)$. In this case, $A_{t}$ is a reason for $B_{t^{\prime}}$, and given $C_{t^{*}}$ it is not. Intuitively, however, $A_{t}$ is not an indirect cause of $B_{t^{\prime}}$ because of $C_{t *}-$ contrary to (8). Similarly, $C_{t *}$ is a reason for $B_{t^{\prime}}$, and given $A_{t}$ it is not. Intuitively, however, $C_{t *}$ is not a spurious cause of $B_{t^{\prime}}$ because of $A_{t}$-contrary to (7). Rather, $C_{t *}$ is a cause of $B_{t^{\prime}}$, and $A_{t}$ is a pre-empted cause, i.e., not a cause of $B_{t^{\prime}}$. But we may easily apply the solution of Lewis (1973b), p. 191: It is also a fact of the case that our poor traveller has not drunk anything for several days $\left(D_{t^{\prime \prime}}, t^{*}<t^{\prime \prime}<t^{\prime}\right)$. Now, $C_{t *}$ is prima facie a cause of $D_{t}$, and $D_{t}$ is prima facie a cause of $B_{t^{\prime}}$; but $A_{t}$ is neither a prima facie, nor a hidden cause of $D_{t}$, and may thus be shown not to be involved in the causal chain leading to $B_{t^{\prime}}$.

Of course, we should scrutinize such examples more carefully. But let us not dwell upon this point; let us rather go on on the assumption that (7) -(9) correctly and completely describe the ways in which causes and prima facie causes may differ. Does this assumption help us in finding an explication of causation, which, to be sure, is not yet given by (7)-(9)? It does, I think. For, the essential point about (7)-(9) is that they develop a very interesting interplay:

Let us assume that $A_{t}$ is prima facie a cause of $B_{t^{\prime}}$ (it will become apparent that we could just as well start with the assumption that $A_{t}$ is prima facie not a cause of $\left.B_{t^{\prime}}\right)$. There may then be a proposition $C_{t_{1}}^{1}\left(t_{1}<t\right)$, because of which $A_{t}$ is secunda facie a spurious, i.e., not a cause of $B_{t^{\prime}}$. But 
there may be a further true proposition $C_{t_{2}}^{2}\left(t_{2}<t\right)$ such that, given $C_{t_{1}}^{1}$ and $C_{t_{2}}^{2}, A_{t}$ is a reason for $B_{t^{\prime}}$. Thus, $A_{t}$ is tertia facie a hidden cause of $B_{t^{\prime}}$ because of $C_{t_{2}}^{1} \cap C_{t_{2}}^{1}$. Still, there may be a third proposition $C_{t_{3}}^{3}\left(t_{3}<t\right)$, which again brings forward the case for spurious causes. And so on. When will this jumping to and fro end? In reality probably rather soon, though one may find examples where the truth is very well hidden. From a logical point of view, however, the to and fro is guaranteed to end only when no true instantaneous proposition before $t$, because of which $A_{\mathrm{t}}$ may turn out to be a spurious or hidden cause, is left unconsidered.

Thus assume that, on the basis of all true instantaneous propositions before $t, A_{t}$ seems to be a cause of $B_{t^{\prime}}$. (Again, we could just as well assume the contrary.) We cannot then prove $A_{t}$ to be spurious, but there may be a true $D_{s_{1}}\left(t<s_{1}<t^{\prime}\right)$ because of which $A_{t}$ is only an indirect cause of $B_{t^{\prime}}$. This still allows the possibility that there is another true $D_{s_{2}}\left(t<s_{2}<t^{\prime}\right)$ suggesting that $A_{t}$ is a hidden direct cause of $B_{t^{\prime}}$. And so on, as before. Again, it is clear that this second to and fro is guaranteed to end only when no true instantaneous proposition between $t$ and $t^{\prime}$ which could continue the to and fro is left unconsidered. Hence, the final decision whether $A_{t}$ is a direct cause of $B_{t^{\prime}}$ is determined by whether $A_{t}$ is a reason for $B_{t^{\prime}}$ on the basis of all true instantaneous propositions before $t$ and between $t$ and $t^{\prime}$. We may turn this into a definition:

(10) Let $w_{0} \in W$ be the actual world, $t<t^{\prime}$, and $Z_{w_{0}, t, t^{\prime}}=\{w \mid w$ is the same as $w_{0}$ for all times $t^{*}$ with $t^{*} \neq t$ and $\left.t^{*}<t^{\prime}\right\} .{ }^{39}$ Then $A_{t}$ is a direct cause of $B_{t^{\prime}}$ in $w_{0}$ iff

(i) $w_{0} \in A_{t} \cap B_{t^{\prime}}$,

(ii) given $Z_{w_{0}, t, t^{\prime}}, A_{t}$ raises the modal rank of $B_{t^{\prime}}$, i.e.:

(iid) $G\left(B_{t^{\prime}} \mid A_{t} \cap Z_{w_{0}, t, t^{\prime}}\right)$, but not $G\left(B_{t^{\prime}} \mid \bar{A}_{t^{\prime}} \cap Z_{w_{0}, t, t^{\prime}}\right)$, and/or $G\left(\bar{B}_{t^{\prime}} \mid \bar{A}_{t} \cap Z_{w_{0}, t, t^{\prime}}\right)$, but not $G\left(\bar{B}_{t^{\prime}} \mid A_{t^{\prime}} \cap Z_{w_{0}, t, t^{\prime}}\right)$, or

(iip) $P\left(B_{t^{\prime}} \mid A_{t} \cap Z_{w_{0}, t, t^{\prime}}\right)>P\left(B_{t^{\prime}} \mid \bar{A}_{t} \cap Z_{w_{0}, t, t^{\prime}}\right)$.

Having thus finished the main line of thought of this paper, the line from (3d) to (10), I wish to add only some miscellaneous, but needed remarks about (10), their point being, of course, to convince you that our end point is a good starting point for going on.

(a) First, I should point out that there are two technical assumptions 
hidden in (10) without which (10) could not be correct. We have tacitly assumed that there are no true instantaneous propositions referring to the time $t$ other than $A_{t}$. If there were such propositions, they should of course be conjoined to. $Z_{w_{0}, t, t}$; but then we would run into the problem of separating $A_{t}$ from these other true propositions referring to $t$. This problem must and can be cleared up in a technically neater account than ours. Moreover, we have tacitly assumed that for each time $t^{\prime}$ there are only finitely many possible pasts of $t^{\prime}$; otherwise, $Z_{w_{0}, t, t^{\prime}}$ is likely to have probability 0 , in which case clause (iip) is meaningless. It is by no means a trivial technical problem to generalize (10) to richer possible worlds, in particular to worlds with a continuous time.

(b) We can now explain the last remark of section 2, i.e., why it is important that the first line of (10), (iid), reads " $G\left(B_{t^{\prime}} \mid A_{t} \cap Z_{w_{0}, t, t t^{\prime}}\right)$, but not $G\left(B_{t^{\prime}} \mid \bar{A}_{t} \cap Z_{w_{0}, t, t^{\prime}}\right)$ " instead of " $G\left(B_{t^{\prime}} \mid A_{t} \cap Z_{w_{0}, t, t^{\prime}}\right)$, but not $G\left(B_{t^{\prime}} \mid\right.$ $\left.Z_{w_{0}, t, t^{\prime}}\right)^{\prime}$ ": Let us split up $Z_{w_{0}, t, t^{\prime}}$ into $Y$ which describes all that has happened in $w_{0}$ up to $t$ and $Y^{\prime}$ which describes all that has happened in $w_{0}$ between $t$ and $t^{\prime}$; thus, $Z_{w_{0}, t, r^{\prime}}=Y \cap Y^{\prime}$. It will be usually the case that $A_{t}$ is determined by its past in $w_{0}$, i.e., that $G\left(A_{t} \mid Y\right)$ is true; and it will be usually the case that $Y^{\prime}$ is compatible with its past in $w_{0}$, i.e., that $G\left(\bar{Y}^{\prime} \mid A_{t}\right.$ $\cap Y)$ is false. However, according to (2), $G\left(A_{t} \mid Y\right)$, not $G\left(\bar{Y}^{\prime} \mid A_{t} \cap Y\right)$, and $G\left(B_{t^{\prime}} \mid A_{t} \cap Y \cap Y^{\prime}\right)$ together imply that $G\left(B_{t^{\prime}} \mid Y \cap Y^{\prime}\right)$. Thus, the second reading is usually unsatisfiable, and we are correct in replacing it by the first reading which has no such problems. By the way, it is very conspicuous here, that causal statements often are counternomological, because $\bar{A}_{t} \cap$ $Z_{w_{0}, t, t *}$ will often describe a counternomological process.

(c) One may wonder, whether it is really possible, as we have apparently assumed in (10), that a direct cause is temporally separated from its direct effect. In a sense, this is possible. Our reluctance to accept this possibility seems to rest on our conviction that causal chains are continuous or gapless. ${ }^{40}$ But this conviction is not an analytical truth. Thus, I think, $(10)$ is not incorrect in allowing temporal gaps between direct causes and their direct effects as a conceptual possibility; we might then use (10) for saying precisely what is characteristic of worlds without such gaps. ${ }^{41}$

(d) It is very clear that (10) can at best be a starting point for a more elaborate theory of causality. This is true all the more so, since we have, in arriving at (10), somewhat secretly narrowed down our topic from causes in general to direct causes only. However, the restoration of the former 
generality of section 2 is a difficult enough task. ${ }^{42}$ So, let us refrain from speculating about such elaborations and confine ourselves to listing a number of heuristic reasons why (10) seems to be a suitable starting point. (Though, of course, the best reason, and in fact the only one that counts in the end, would be that (10) is part of a satisfying, fully elaborated theory of causality.)

(e) A very important aspect of the acceptability of an explication, the evaluation of which is, however, rather subjective, is to what extent we can grasp the concepts of the explicans independently of the explicandum. The status of the regularity theory of causality has never been very clear in this respect, because the question, which regularities are suitable for the regularity theory, still threatens to set us back to causal concepts. (3d) in itself, for instance, was quite worthless because of this aspect; and backing up counterfactual conditionals by a realistic similarity semantics, as is done in Lewis (1973a), is not very helpful for the same reason. ${ }^{43}$ I think, (10) fares better, if we interpret conditional relations and probability measures epistemically, because this interpretation seems to me to be well understandable without recourse to the causal concepts to be explicated. Whether this advantage can be transferred to the realistic interpretation, is, however, unclear, since this interpretation is unsettled.

(f) Independent confirmation for (10) is contributed from an unexpected side, from statistics. Currently the most discussed (and perhaps the most favored) explication of probabilistic causality among statisticians is the one worked out by Granger. If one compares (10p) with the explication stated in Granger (1980), p. 330 and p. 336f., one immediately discovers a striking similarity. ${ }^{44}$

(g) Of course, (10) is reasonable to the extent to which this section's considerations leading to (10) were reasonable and truly reflected the differences between reasons and causes. But we still have to compare sections 3 and 4 . The differences between (5) and (10) are obvious: (10) restricts itself to direct causes and puts the unambiguous $Z_{w_{0}, t, t^{\prime}}$ in the place of the obscure obtaining circumstances. Have we thereby evaded the two problems of circularity which threatened the explication of the obtaining circumstances?

Concerning the first circularity, we have done just the thing we formerly claimed we shouldn't be doing; that is, a lot of instantaneous propositions causally irrelevant to the $A_{t}$ and $B_{t^{\prime}}$ of (10) may, and normally will, be 
conjoined in the $Z_{w_{\text {n.t. }} t^{\prime}}$ of (10). But the following procedure shows doing this to be harmless: We proceed from (10) to an explication of causal irrelevance, and then we prove that the definition (10) is equivalent to a definition obtained from (10) by replacing $Z_{w_{0}, t, t}$ by the proposition which is the conjunction of all and only the instantaneous, true propositions causally relevant to $A_{t}$ and $B_{t^{\prime}}$. However, this is a topic for another, more formal paper.

The second circularity is avoided simply by restricting (10) to direct causes. It was no accident that we could use the same example for demonstrating the second circle as for illustrating the case (8) of indirect causes. This shows that this circularity applies only to indirect causes and that the $Z_{w_{0}, t, t *}$ of (10) is, thus, not incorrect in conjoining all true instantaneous propositions between $A_{t}$ and $B_{t^{\prime}}$. However, like the first, the avoidance of the second circularity will also be complete only when a satisfying explication of indirect causes is joined to (10).

(z) Last and least, we may observe that (10) is indeed nothing but a precise version of our preliminary picture in section 1 . If that picture pleased you, (10) might also please you.

\section{Universität München}

\section{NOTES}

1 Actually, Stegmüller is very cautious not to draw this conclusion. Rather, he says that it is a matter of stipulation whether to explicate explinations such that Vernunftgründe are sufficient for them or not. But it is all too apparent which explication he favours.

2 Not a reason to do something; we are here not concerned with practical matters.

${ }^{3}$ For instance, an important criticism of Hempel's theory of explanation has been that his theory of deductive-nomological explanation and his theory of inductive-statistical explanation seem to be disparate. Cf. Stegmüller (1983), ch. XI.

${ }^{4}$ Though (1) is not impeccable, of course. A minor question is whether $A$ 's being a reason for $B$ for $X$ implies that $A$ is in fact believed by $X$. According to (1), this is not implied. But, one may as well define the matter in such a way that it is implied. A more serious problem is posed by the fact that there are non-reasonable ways for beliefs to raise other beliefs. The belief that my second alarm is ringing makes me jump out of my bed and, as it happens, stumble over a book on the floor and thus believe that there is a book on the floor. But, certainly, the ringing of my second alarm is not a reason for me to believe that there is a book on the floor. However, this problem of "deviant causal chains" is beyond the scope of this paper; cf. Peacocke (1979).

${ }^{5}$ Cf. Lewis (1973a), sect. 4.1.

6 That's the reason why "possible world" is misleading. Nothing is more natural to assume than that, whatever possible worlds might be, they are such that our real world is one possible 
world. But precisely this is wrong. According to the above, common understanding the real world is not a possible world!

7 In particular in (1967).

${ }^{8}$ Cf. Davidson (1967), p. 85f., or Mackie (1974), pp. $250 \mathrm{ff}$.

9 Thus, propositions de dicto are what is usually meant by propositions simpliciter. And the proposition de re expressed by a sentence is the usual proposition which that sentence expresses, if all its singular terms are taken as rigid designators (even if they are not). I find the term "proposition de re" not quite happy, but there does not seem to be an established term for it.

10 According to Davidson (1969), these are the next to the most correct identity conditions for events. And they give us a good notion of what events are to be, whereas the most correct identity conditions - namely that two events are identical iff they have exactly the same causes and effects - are not very helpful in this respect, as Davidson himself acknowledges.

11 There seem to be exceptions, e.g. Kim (1971) or Lewis (1973b). But what Kim (1971), sect. IV, defines as events are not events as just explained, but rather something like our propositions de re. And Lewis (1973b) is a very special case, because he has quite a different notion of possible worlds and thus of propositions than we have. This allows him a sort of identification of events with certain propostions in his sense, which I can only find dubious; cf. Lewis (1973b), p. 186. However, there is a better way of bringing together events and propositons in our sense. Let us make the minimally materialistic assumption that all properties of an object are supervenient upon its basic physical properties (for the concept of supervenience see Kim (1978)). Then we may identify an event with a complete basic description of the space-time region occupied by this event, because, according to this supervenience assumption, all the possibly indescribably many other things true of that region are already uniquely determined by this basic description. But now, we have made events propositional, so to speak, and not propositions eventual, as Lewis did. Therefore, if we accept this way of identification, propositions keep their role of primary causal objects, and events may become causal objects only by way of this identification.

12 One may drop the requirements of closure under logical entailment, as does, e.g., Ellis (1979). But this would be pointless for this paper. Moreover, by taking propositions as $X$ 's epistemic objects, we have already assumed $X$ to be logically so perfect as to recognize sentences expressing the same proposition.

${ }^{13}$ Obviously, we have just twice presupposed that $W$ is not too large. If $W$ is very large, probabilities may not be definable for all subsets of $W$. We must then also take care to distinguish between entailment by finitely and infinitely many premises. However, such niceties will not be considered here.

${ }^{14}$ There are essentially three ways of reducing or closing this gap. One may make appropriate regularity requirements, as Carnap (1971), sect. 7, and (1980), sect. 21, has proposed, in order to minimize the number of null propositions. One may go over to Renyi or Popper measures; cf. van Fraassen (1976). Or one may delve into non-standard measure theory; $\mathrm{cf}$. Loeb (1979).

15 Some readers may have expected to read " $P_{X . s}(B)$ " instead of " $P_{X, s}(B \mid \bar{A})$ ". However, this clearly makes no difference here. My reasons for this formulation will soon become apparent.

16 The term is borrowed from Lewis (1973a), sect. 2.7.

17 As I have said above this is now the standard account of conditionalization within a deterministic framework. By this I am referring to the fact that (2) may be said to be the (simplified or simplistic) common core of the existing semantics for a logic of conditional belief and the existing epistemic interpretations for a logic of conditionals; cf., e.g., von Kutschera (1976), ch. 4, Ellis (1979), ch. III, or Gärdenfors (1981). However, a more detailed 
comparison of (2) with these semantics and interpretations is not relevant for our purpose. ${ }_{18}$ Still, (1d) is by no means perfect. One of the most apparent flaws is that according to (1d) one can already believe a reason and what it is a reason for (because we have taken (i) instead of (iii)), but one cannot get further (not yet believed) reasons for something already believed. I find this counterintuitive. However, the standard accounts referred to in note 17 do not help here, and improving upon them is much beyond our scope. In this paper, we may be content with (1d).

19 If this claim seems too strong for you, take it as expressing my intention to focus on the counterfactual analysis. Von Wright (1974), e.g., would be opposed to the claim that this is the only promising approach besides the regularity theory; and Beauchamp, Rosenberg (1981) would deny that the regularity theory has declined. But so far I have not found convincing answers by regularity theorists to the problems mentioned by Lewis (1973b), p. 180f., or Mackie (1974), ch. 3. In particular, the difficulty presented by Mackie (1974), pp. $81-87$, seems to me to be insurmountable. Mackie there describes two situations which clearly differ in counterfactual and causal structure, but which nevertheless have the same nomological structure and thus can't be distinguished by any regularity account. The deeper reason behind such defeats of the regularity theory is the fact that causal counterfactuals often are counternomologicals, in which case the invocation of the true laws is not helpful. See also remark (b) of section 4.

${ }^{20}$ This statement has the number (3d), because it clearly falls into the deterministic framework.

21 In cases of symmetric or asymmetric causal overdetermination, however, a difference between causal and the corresponding counterfactual statements emerges. But we shall not go into these cases. Cf. Lewis (1973b), p. 191, and Loeb (1974).

${ }^{22}$ This need not be a physical point of time. It is a point of time of our possible worlds in $W$ which may have a coarser time than physical time.

${ }^{23}$ Cf. the impressive list of philosophers entertaining (4d) given by Sosa (1975), p. 1.

${ }^{24}$ Cf. Sosa (1975), p. 1f., or Mackie (1974), ch. 2, a good deal of which is about this point. 25 This second circle is of course analogous to the one concerning contenability. Cf. Goodman (1965), part I, 2.

26 It may aiso be pointed out that statisticians are discussing causality as vigorously as philosophers are, and surely, their subject is probabilistic causality. Cf. p. 332, p. 337, and the references of Granger (1980).

27 From premises consistent with the laws, to be precise. The second line of the definition of $G_{L}$ secures that $G_{L}$ satisfies clause (i) of (2).

${ }^{28}$ Cf., e.g., Lewis (1973a) and Pollock (1976). However, the " $G$ " of (5d) should not be exactly equated with such a conditional. The reason is that a statement of the form "if $C$ would be (or had been) the case, then $D$ would be (or have been) the case" does, as clause (ii) of (3d), implicitly assume the (cotenable) obtaining circumstances as given, which may therefore be arbitrarily conjoined to the antecedens of that statement without changing its truth-value (cf. Lewis (1973a), p. 57). In contrast to this, it makes a difference for a statement of the form " $G(D \mid C)$ " how many of the obtaining circumstances are conjoined to $C$. In a nutshell, whereas conditionals are meant to capture the whole factual and counterfactual structure of our world (and also of possible worlds), the $G$ of (5d) is intended to capture only the nomological and counternomological structure of our world.

29 Thereby, I do not mean that it is often difficult to decide whether such a statement is true or false. I mean that we often do not know what could be meant by calling such a statement true or false.

30 That is, with an epistemically interpreted conditional relation instead of his subjective 
confirmation function $C$ and another realistically interpreted conditional relation instead of his chance function $P$.

31 Therefore I doubt that such a rewriting would please Lewis. In (1980), he confesses to be a subjectivist not denying objective chance, but thinking that objective chance can be best or only understood from a subjectivistic point of view. But this is not his stance towards counterfactuals, which seems to be essentially unchanged since his (1973a). Though he does not deny the feasibility of an epistemic interpretation, there is no word about the implied thesis that the realistic interpretation of conditionals is only or best understood through the epistemic interpretation.

32 In particular the answer "in no sense and to no extent", which has again been argued to be wrong in our days by Putnam (1980) and (1981), ch. 1-3.

${ }^{33}$ Suppes' definition of prima facie causes in (1970), p. 12, is almost identical with (6p). There is a small difference: Suppes does not require $A_{t}$ and $B_{t^{\prime}}$ to obtain. On p. 40 he explains that in all his definitions he has explicated only potential causal relations which are actual, if the propositions standing in these relations are actual, i.e., true. I feel it is a bit less confusing to concentrate only on what Suppes calls actual causal relations.

${ }^{34}$ Suppes (1970) takes into account only the first two ways, i.e., that prima facie causes may be spurious or indirect causes. In Spohn (1980), I have explored, within the probabilistic framework, some consequences of the third way, i.e., of the fact that causes may also be hidden. The following generalizes (part of) this to the deterministic framework.

35 The "only then" is important. If we could not find such a $C_{t *}$, because of which $A_{t}$ is a spurious cause of $B_{t^{\prime}}$, we would grant that $A_{t}$ really is a cause of $B_{t^{\prime}}$.

${ }^{36}$ Again, if we could not find such a $C_{t *}$ mediating the causal chain from $A_{t}$ to $B_{t^{\prime}}$, we would grant that $A_{t}$ is a direct cause of $B_{t^{\prime}}$.

${ }_{37}$ Once more: if we could not find such a $C_{t *}$ because of which $A_{t}$ is a hidden cause of $B_{t^{\prime}}$, we would stick to the opinion that $A_{t}$ is not a cause of $B_{t^{\prime}}$.

38 Cf. e.g. Mackie (1974), p. $44 f$.

39 This amounts to the idea that $Z_{w_{0}, t, t^{\prime}}$ is the conjunction of all instantaneous, (in $w_{0}$ ) true propositions before $t$ and (strictly) between $t$ and $t^{\prime}$.

${ }^{40}$ If time is not continuous in the possible worlds in $W$, causal chains can't be neither. Here, I intend the gaplessness of causal chains to mean the analogue to continuity within discontinuous time.

${ }^{41}$ It will be no surprise that, within the probabilistic framework, there is a close connection between such gaplessness and Markov chains. Indeed, it is often said that Markov chains are characterized by the "absence of after-effect". Cf. Spohn (1980), p. 91f.

42 One idea is to say that the relation of being a cause simply is the transitive closure of the relation of being a direct cause; and an indirect cause is then a cause which is not direct. This is plausible, but it faces three problems. First, this idea cannot be applied, if the possible worlds in $W$ have continuous time. This is so because in this case the relation of being a direct cause is likely to be empty or to be confined to simultaneous instantaneous propositions, and the transitive closure would then be empty or so confined as well, which is not what we want. Secondly, this idea seems to be inadequate for the probabilistic framework, because there it is rather doubtful that the relation of being a cause is transitive; cf. Suppes (1970), p. 58f. Thirdly, another idea would be that, just as we have arrived at (10) by maximally exploiting (7) - (9), we may exploit (7)-(9) for an explication of indirect causes. I have tried this idea in Spohn (1980) within the probabilistic framework. The problem is that it is not at all clear that the two ideas are consonant even within the deterministic framework. ${ }^{43}$ In (1979), pp. 465 467, Lewis expressly acknowledges that similarity semantics does not provide an independent grasp of counterfactuals; rather, he says, the similarities between 
possible worlds inferred from true or accepted counterfactuals only help us in systematizing all counterfactuals we may consider.

${ }^{44}$ In Spohn (1983), I have carried out this comparison a bit more carefully.

\section{REFERENCES}

Beauchamp, T.L., A. Rosenberg: 1981, Hume and the Problem of Causation, Oxford: University Press.

Carnap, R.: 1971, 1980, 'A Basic System of Inductive Logic'. Part I in: R. Carnap, R.C. Jeffrey (eds.), Studies in Inductive Logic and Probability, vol. I, Berkeley: University Press, 1971. Part II in: R.C. Jeffrey (ed.), Studies in Inductive Logic and Probability, vol. II, Berkeley: University Press, 1980.

Davidson, D.: 1967, 'Causal Relations', Journal of Philosophy 64 (1967), 691-703. Also in Sosa (1975), pp. 82-94. Quotations refer to the latter printing.

Davidson, D.: 1969, 'The Individuation of Events', in N. Rescher (ed.), Essays in Honor of Carl G. Hempel, Dordrecht: D. Reidel, 1969, pp. 216-234.

Ellis, B.: 1979, Rational Belief Systems, Oxford: Blackwell.

van Fraassen, B.C.: 1976, 'Representation of Conditional Probabilities', Journal of Philosophical Logic 5, 417-430.

Gärdenfors, P.: 1981, 'An Epistemic Approach to Conditionals', American Philosophical Quarterly 18, 203-211.

van de Geer, J.P.: 1971, Introduction to Multivariate Analysis for the Social Sciences, San Francisco: Freeman.

Goodman, N.: 1965, Fact, Fiction, and Forecast, Indianapolis: Bobbs-Merrill, 2nd edition.

Granger, C.W.J.: 1980, 'Testing for Causality. A Personal Viewpoint', Journal of Economic Dynamics and Control 2, 329-352.

Hempel, C.G.: 1965, Aspects of Scientific Explanation and Other Essays in the Philosophy of Science, New York: Free Press.

Kim, J.: 1971, 'Causes and Events: Mackie on Causation', Journal of Philosophy 68, 426441.

Kim, J.: 1978, 'Supervenience and Nomological Incommensurables', American Philosophical Quarterly 15, 149-156.

von Kutschera, F.: 1976, Einführung in die intensionale Semantik, Berlin: de Gruyter.

Lewis, D.: 1973a, Counterfactuals, Oxford: Blackwell.

Lewis, D.: 1973b, 'Causation', Journal of Philosophy 70 (1973), 556-567. Also in Sosa (1975), pp. 180-191. Quotations refer to the latter printing.

Lewis, D.: 1979, 'Counterfactual Dependence and Time's Arrow', Noús 13, 455-476.

Lewis, D.: 1980, 'A Subjectivist's Guide to Objective Chance', in R.C. Jeffrey (ed.), Studies in Inductive Logic and Probability, vol. II, Berkeley: University Press, pp. 263-293.

Loeb, L.E.: 1974, 'Causal Theories and Causal Overdetermination', Journal of Philosophy 71, 525-544.

Loeb, P.A.: 1979, 'An Introduction to Nonstandard Analysis and Hyperfinite Probability Theory',in: A. T. Bharucha-Reid (ed.), Probabilistic Analysis and Related Topics, vol. 2. New York: Academic Press, pp. 105-142.

Mackie, J.L.: 1974, The Cement of Universe, Oxford: University Press.

Peacocke, C.: 1979, 'Deviant Causal Chains', in P.A. French, T.E. Uehling, H.K. Wettstein (eds.), Midwest Studies in Philosophy, vol. IV, Studies in Metaphysics, Minneapolis: University of Minnesota Press, pp. 123-155.

Pollock, J.L.: 1976, Subjunctive Reasoning, Dordrecht: D. Reidel. 
Putnam, H.: 1980, 'Models and Reality', Journal of Symbolic Logic 45, 464-482.

Putnam, H.: 1981, Reason, Truth and History, Cambridge: University Press.

Sosa, E. (ed.): 1975, Causation and Conditionals, Oxford: University Press.

Spohn, W.: 1980, 'Stochastic Independence, Causal Independence, and Shieldability', Journal of Philosophical Logic 9, 73-99.

Spohn, W.: 1983, 'Probabilistic Causality: From Hume via Suppes to Granger', in G. Gambetta, M.C. Galavotti (eds.), Causalita' e Modelli Probabilistici, Bologna: Cooperativa Libraria Universitaria Editrice, forthcoming.

Stegmüller, W.: 1969, 1983, Probleme und Resultate der Wissenschaftstheorie und Analytischen Philosophie, Band I, Wissenschaftliche Erklärung und Begründung, Berlin: Springer, 1969, 2nd revised edition 1983.

Suppes, P.: 1970, A Probabilistic Theory of Causality, Amsterdam: North-Holland.

von Wright, G.H.: 1974, Causality and Determinism, New York: Columbia University Press.

Manuscript received 3 November 1982. 